\title{
(1) Festveranstaltung anlässlich des 100. Geburtstags der DGP
}

Die DGP feierte am 6. Oktober 2010 ihren 100. Geburtstag mit einer offiziellen Festveranstaltung in Berlin. Anfang des Jahres war bereits die DGP-Chronik „100 Jahre DGP - 100 Jahre deutsche Pneumologie“ erschienen und an alle Mitglieder verteilt worden.

Die Festveranstaltung fand im historischen Langenbeck-Virchow-Haus statt und war mit 150 Teilnehmern gut besucht ( $\bullet$ Abb. 1), darunter mehrere Past-Präsidenten der Gesellschaft, Ehrenmitglieder, Korrespondierende Mitglieder und Leiter der verschiedenen Sektionen der DGP. Im Rahmen der Festveranstaltung wurden Grußadressen von Prof. Dr. Claus Vogelmeier, Präsident der DGP, Prof. Dr. Hendrik Lehnert, Präsident der Deutschen Gesellschaft für Innere Medizin $(\checkmark$ Abb. 2) und Prof. Dr. Marc Decramer, Präsident

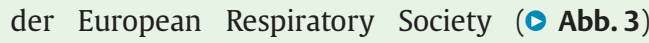
übermittelt. Alle Redner hoben die inzwischen gewachsene nationale und internationale Bedeutung der DGP hervor.

Ein Highlight der Veranstaltung war der Vortrag von Prof. Dr. Fritz Beske ( $\bullet$ Abb.4) vom Fritz Beske-Institut für Gesundheits-System-Forschung in Kiel zum Thema „Bedeutung der Bevölkerungsentwicklung und der Morbiditätsentwicklung für die Gesundheitsversorgung von morgen“. Er befasste sich darin kritisch mit der zukünftig zu erwartenden Entwicklung des Gesundheitswesens und stellte eigene Lösungsvorschläge vor.
Den Festvortrag hielt Prof. Dr. h.c. mult. Ewald Weibel aus Bern/Schweiz ( $\bullet$ Abb.5) mit dem Thema „Der guten Lunge guter Bau - die morphometrischen Grundlagen der Lungenfunktion“. Er ging in dem Zusammenhang auch speziell auf das Geburtsjahr 1910 ein, in dem für die Pneumologie bahnbrechende Forschungen von Nils Bohr und August Krogh veröffentlicht worden waren. Zuvor erhielt Ewald Weibel die Urkunde zur Ernennung zum Korrespondierenden Mitglied der DGP vom Präsidenten überreicht ( $\bullet$ Abb. 6 ). Die DGP drückte damit ihre große Wertschätzung für seine herausragenden Grundlagenforschungen zur Struktur der Lunge aus.

Zum Schluss der Festveranstaltung wurde Prof. Dr. Stefan Andreas von der Lungenfachklinik Immenhausen/Universität Göttingen mit dem erstmalig ausgeschriebenen Oskar und Helene-Medizin-Preis, dotiert mit 50000,00€, für seine Forschungsergebnisse auf dem Gebiet der COPD ausgezeichnet.

Mit Klavierkompositionen aus dem DGP-Gründungsjahr 1910, ausgewählt und vorgetragen von Frau Prof. Linde Großmann von der Universität der Künste in Berlin, der Schwester von Prof. Dr. Detlef Kirsten, fand die Festveranstaltung eine glanzvolle musikalische Umrahmung.

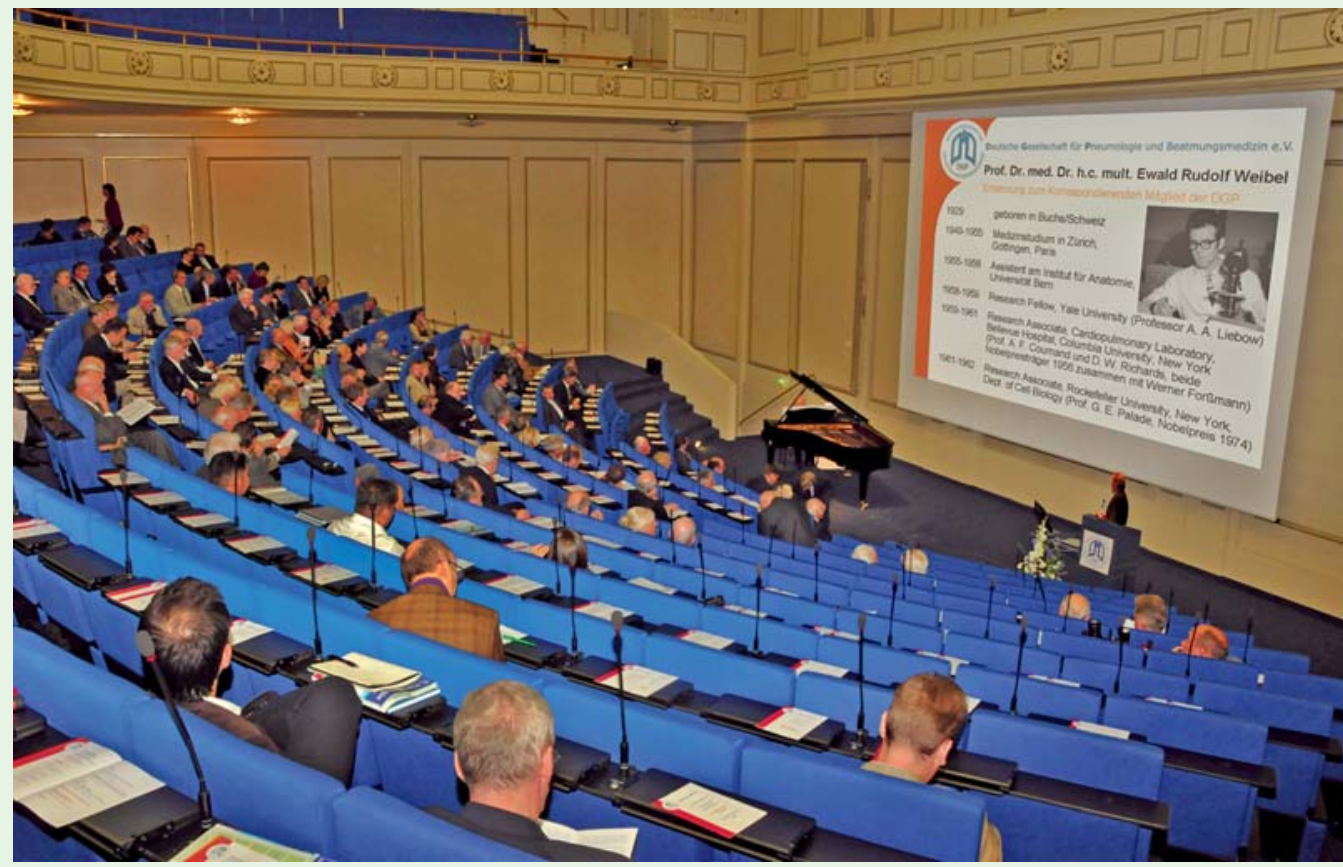

Abb. 1 Blick in den Hörsaal des Langenbeck-Virchow-Hauses. 


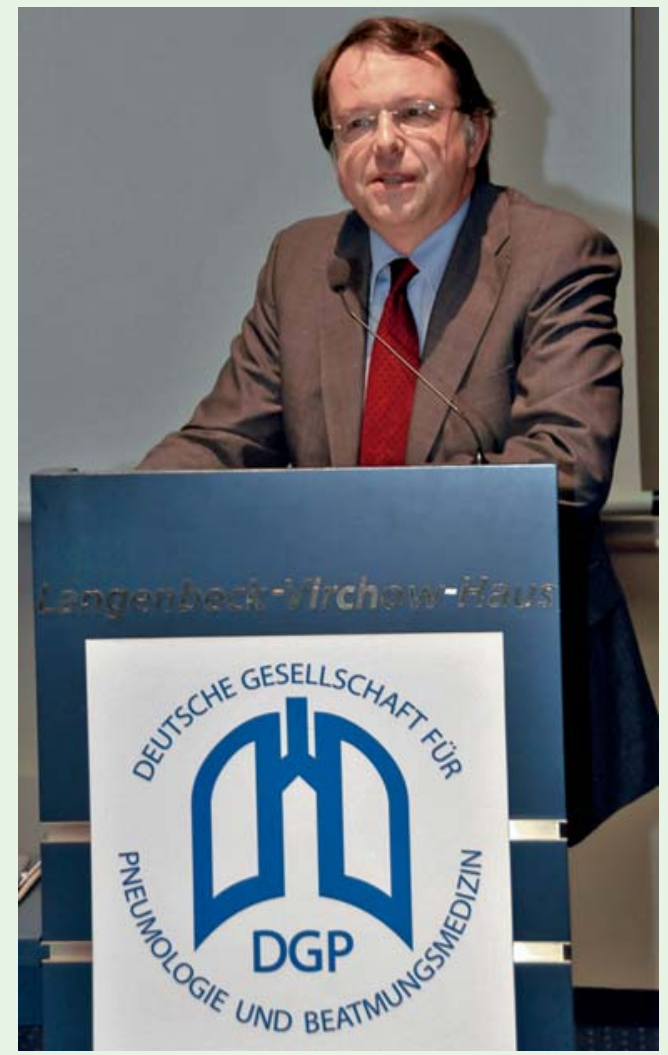

Abb. 2 Prof. Dr. Hendrik Lehnert, Präsident der Deutschen Gesellschaft für Innere Medizin.

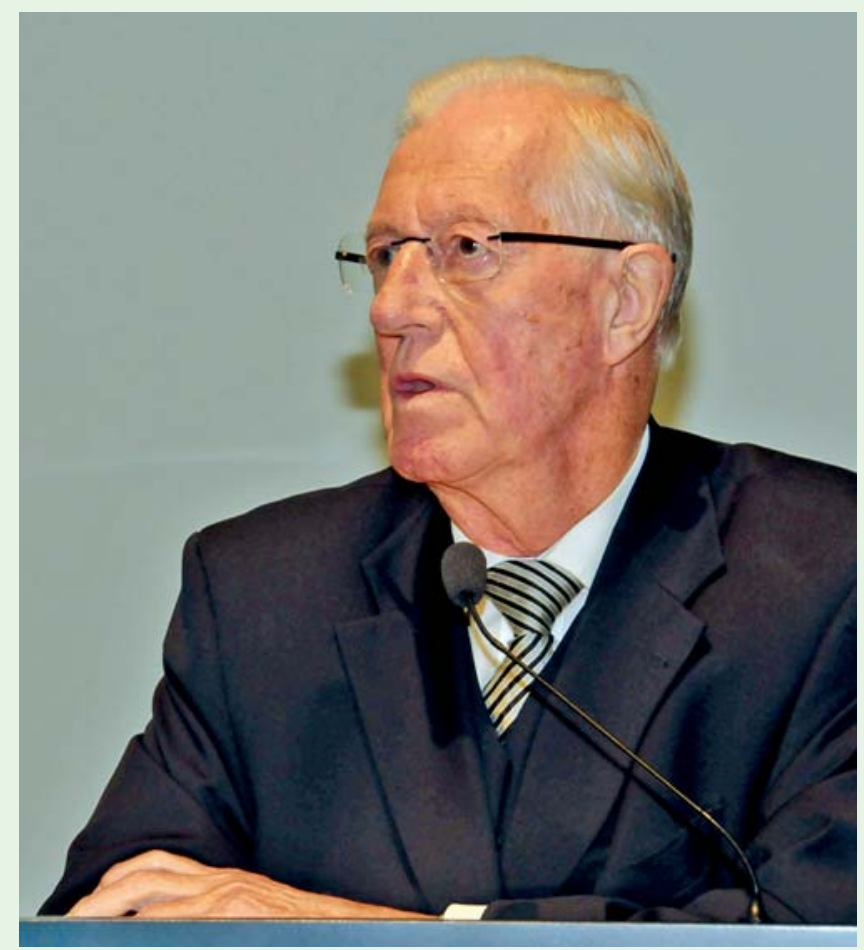

Abb. 4 Prof. Dr. Fritz Beske.

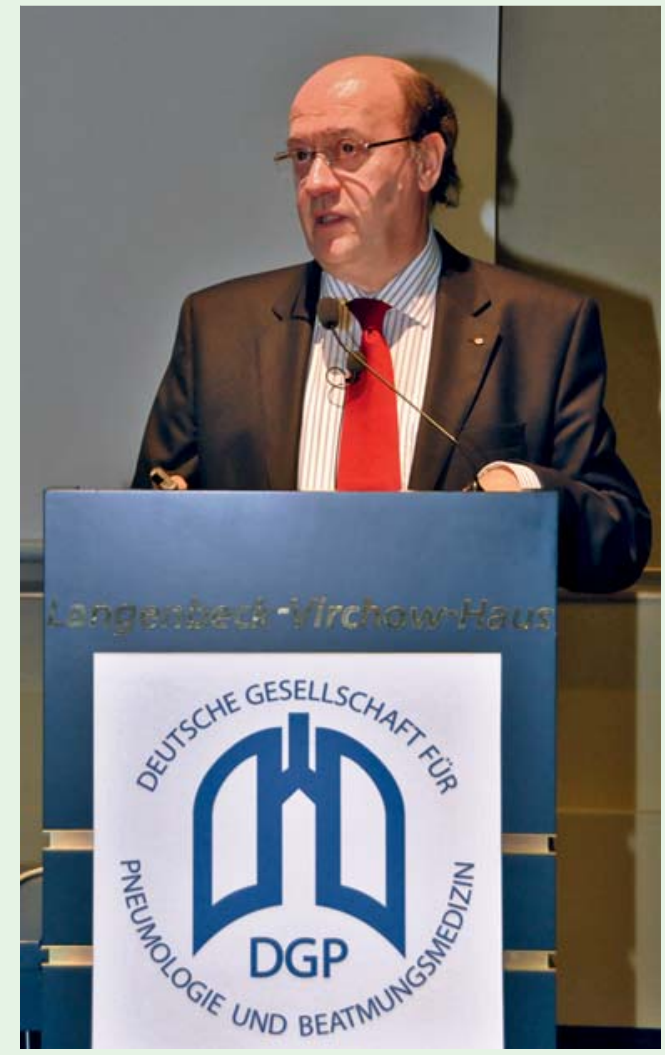

Abb. 3 Prof. Dr. Marc Decramer, Präsident der European Respiratory Society.

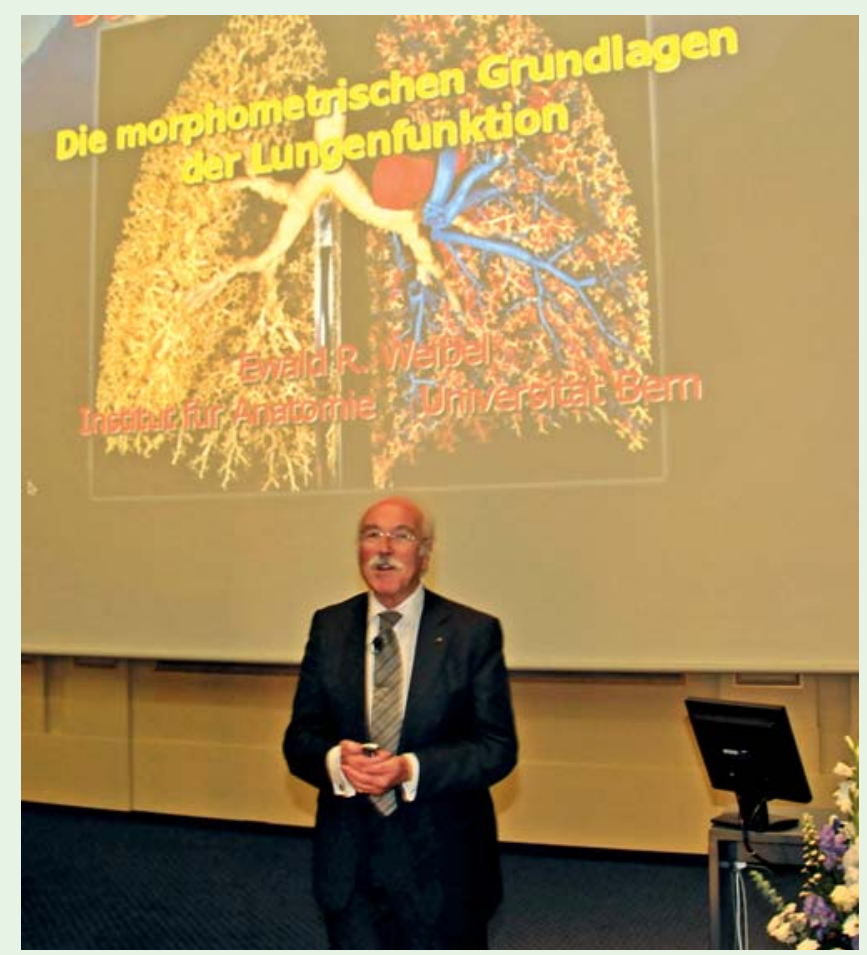

Abb. 5 Prof. Dr. h.c. mult. Ewald Weibel. 


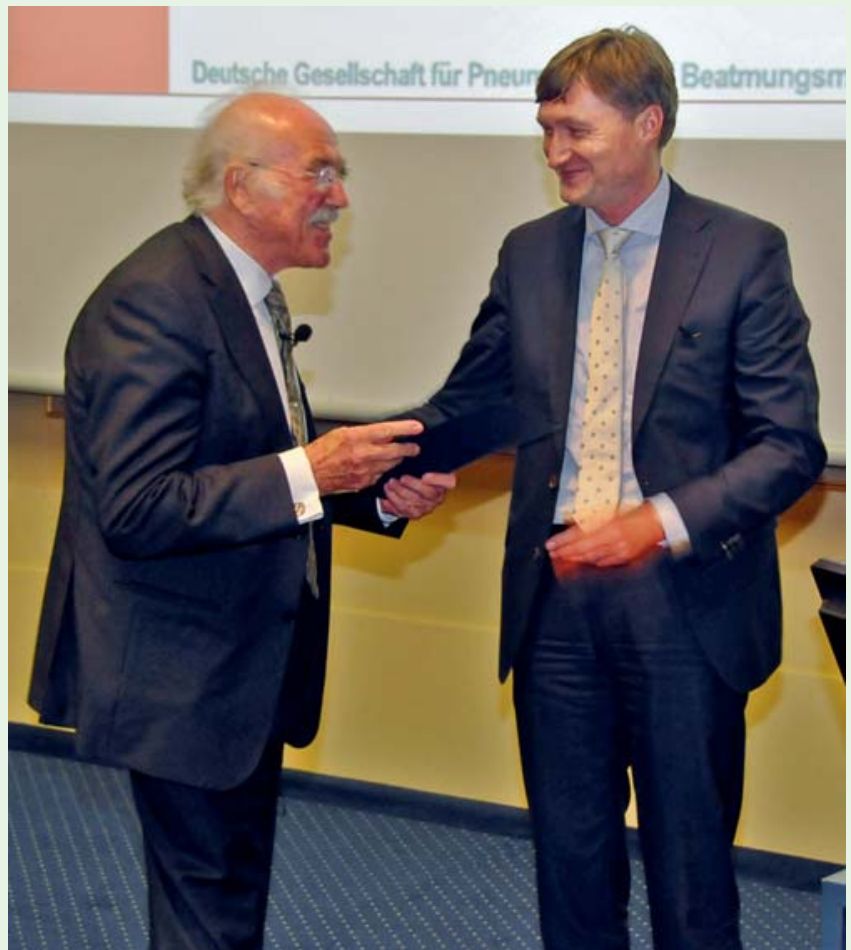

Abb. 6 Überreichung der Urkunde als Korrespondierendes DGP-Mitglied durch den Präsidenten, Prof. Vogelmeier an Prof. Weibel.

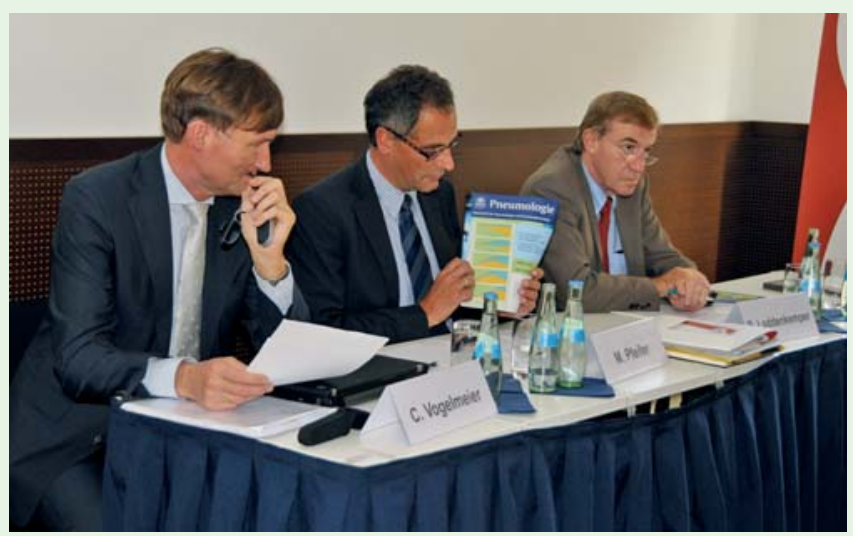

Abb. 7 Pressekonferenz (von links nach rechts: C. Vogelmeier, M. Pfeifer, R. Loddenkemper).
Vor der Veranstaltung fand eine Pressekonferenz zu den wichtigsten Themen der Pneumologie statt, wobei im Mittelpunkt das September-Heft der „Pneumologie“ zu „100 Jahre DGP“ und zum internationalen „Year of the Lung“ stand ( $\bullet$ Abb. 7). In dem Heft findet sich auch ein Artikel aus dem Institut für Krebsepidemiologie an der Universität zu Lübeck und dem Institut für Gesundheitsforschung in Kiel, „Erkrankungszahlen in der Pneumologie - eine Projektion bis 2060“, der zum Teil erhebliche Steigerungen bei den pneumologischen Krankheiten in den nächsten Jahrzehnten prognostiziert. Auf die wichtigsten Krankheitsbilder wurde in der Pressekonferenz hinsichtlich ihrer geschichtlichen Entwicklung, des derzeitigen Status und mit Ausblick auf ihre zukünftige Entwicklung eingegangen. Die einzelnen Beiträge wurden über „Lungenärzte im Netz“ verbreitet. In der „Ärztezeitung“ erschien jeweils ein längerer Übersichtsartikel zur Entwicklung der DGP in den vergangenen 100 Jahren mit dem Titel „Von der Lieghalle zur Heimbeatmung“ sowie zum „Year of the Lung“ mit dem Titel „Asthma, COPD und Co. breiten sich global aus“. Insgesamt war dies eine würdige Jubiläumsfeier, die die Bedeutung der Pneumologie und die Rolle der DGP für die Entwicklung des Faches sichtbar gemacht hat.

\section{R. Loddenkemper}

